\title{
ILLUMINATED CAPS OF THE UNIT SPHERES OF BANACH SPACES AND RELATED INEQUALITIES
}

\author{
Senlin Wu, Xinling Zhang And XinXin Tong
}

Abstract. Geometric properties of illuminated caps of the unit sphere of a finite dimensional Banach space play an important role in Hadwiger's covering problem for centrally symmetric convex bodies. In view of this, fundamental properties of illuminated caps are presented, incenters, inradii, and self-circumradii of illuminated caps are studied, and related inequalities are obtained.

Mathematics subject classification (2010): 46B20.

Keywords and phrases: Illuminated caps, incenter, inradius, self-circumradius.

\section{REFERENCES}

[1] J. Alonso, H. Martini, And Senlin Wu, On Birkhoff orthogonality and isosceles orthogonality in normed linear spaces, Aequat. Math. 83: 153-189, 2012.

[2] K. BEZDEK, Hadwiger's covering conjecture and its relatives, Amer. Math. Monthly 99 (10): 954 956, 1992.

[3] K. BezdeK, Classical Topics in Discrete Geometry, CMS Books in Mathematics/Ouvrages de Mathématiques de la SMC, Springer, New York, 2010.

[4] K. BEZDEK AND M. A. KHAN, The geometry of homothetic covering and illumination, ArXiv eprints, February 2016.

[5] V. Boltyanski, H. Martini, And P. S. Soltan, Excursions into Combinatorial Geometry, Universitext, Springer-Verlag, Berlin, 1997.

[6] I. ŞERB, Rectangular modulus, Birkhoff orthogonality and characterization of inner product spaces, Commentationes Mathematicae Universitatis Carolinae 40 (1): 107-119,1999.

[7] J. GAO AND K. S. LAU, On the geometry of spheres in normed linear spaces, J. Austral. Math. Soc. 48: 101-112, 1990.

[8] H. Hadwiger, Ungelöste Probleme, No. 20. Elem.Math. 12: 121, 1957.

[9] Chan He and Yunan Cui, Some properties concerning Milman's moduli, J. Math. Anal. Appl. 329 (2): 1260-1272, 2007.

[10] M. LASSAK, Solution of Hadwiger's covering problem for centrally symmetric convex bodies in $E^{3}$, J. London Math. Soc. 30 (3): 501-511, 1984.

[11] H. Martini AND V. SOltan, Combinatorial problems on the illumination of convex bodies, Aequat. Math. 57 (2-3): 121-152, 1999.

[12] H. Martini, K. J. SWAnepoel, And G. Weiss, The geometry of Minkowski spaces - a survey, Part I, Expo. Math. 19: 97-142, 2001.

[13] R. E. Megginson, An Introduction to Banach Space Theory, Springer-Verlag, New York, 1998.

[14] R. Webster, Convexity, Oxford University Press, New York, 1994.

[15] SENLIN WU, Upper bounds for the covering number of centrally symmetric convex bodies in $\mathbb{R}^{n}$, Math. Inequal. Appl. 17 (4): 1281-1298, 2014.

[16] Senlin Wu, Xinjian Dong, AND Dan Wang, Circle-uniqueness of pythagorean orthogonality in normed linear spaces, Journal of Function Spaces, 2014: Article Id 634842, 2014. 